\title{
A Natural Higgs Mass in Supersymmetry from Non-Decoupling Effects
}

\author{
Kohsaku Tobioka ${ }^{* \dagger}$ \\ Kavli IPMU \\ E-mail: kohsaku.tobioka@ipmu.jp
}

The Higgs mass implies fine-tuning for minimal theories of weak scale supersymmetry (SUSY). Non-decoupling effects can boost the Higgs mass when new states interact with the Higgs, but new sources of SUSY breaking that accompany such extensions threaten naturalness. We show that two singlets with a Dirac mass can increase the Higgs mass while maintaining naturalness in the presence of large SUSY breaking in the singlet sector. We explore the modified Higgs phenomenology of this scenario, which we call the "Dirac NMSSM."

Proceedings of the Corfu Summer Institute 2014

3-21 September 2014

Corfu, Greece

* Speaker.

${ }^{\dagger}$ A footnote may follow. 


\section{Introduction}

\subsection{Surprises at the $\mathrm{LHC}$}

The discovery of a new resonance at $125 \mathrm{GeV}$ [1], that appears to be the long-sought Higgs boson, marks a great triumph of experimental and theoretical physics. On the other hand, the presence of this light scalar forces us to face the naturalness problem of its mass. Arguably, the best known mechanism to ease the naturalness problem is weak-scale supersymmetry (SUSY), but the lack of experimental signatures is pushing SUSY into a tight corner. In addition, the observed mass of the Higgs boson is higher than what was expected in the Minimal Supersymmetric Standard Model (MSSM), requiring fine-tuning of parameters at the $1 \%$ level or worse [2]. This comes from a fact that it is necessary for the observed Higgs mass to have the large radiative corrections by large stop mass, $m_{\tilde{t}}^{2}$, or by large left-right mixing of stops, $X_{t}$, where the Higgs mass formula is

$$
m_{h, \mathrm{MSSM}}^{2} \simeq m_{Z}^{2} \cos 2 \beta+\frac{3 m_{t}^{4}}{4 \pi^{2} v^{2}}\left(\log \frac{m_{\tilde{t}}^{2}}{m_{t}^{2}}+m_{\tilde{t}}^{2}\left(1-\frac{X_{t}^{2}}{12 m_{\tilde{t}}^{2}}\right)\right),
$$

while these large $m_{\tilde{t}}$ and $A_{t}$ make the theory unnatural because the Higgs soft mass can be very different from the weak scale by a fast Renormalization Group (RG) evolution,

$$
\mu \frac{d}{d \mu} m_{H_{u}}^{2} \simeq \frac{3 y_{t}^{2}}{8 \pi^{2}}\left(2 m_{\tilde{t}}^{2}+\left|A_{t}\right|^{2}\right)
$$

where $A_{t}$ is a soft breaking part of $X_{t}$.

If SUSY is realized in nature, one possibility is to give up on naturalness [3]. Alternatively, theories that retain naturalness must address two problems, (I) the missing superpartners and (II) the Higgs mass. The collider limits on superpartners are highly model-dependent and can be relaxed when superpartners unnecessary for naturalness are taken to be heavy [4], when less missing energy is produced due to a compressed mass spectrum [5] or due to decays to new states [6], and when $R$-parity is violated [7]. Even if superpartners have evaded detection for one of these reasons, we must address the surprisingly heavy Higgs mass.

\subsection{Beyond the MSSM}

There have been many attempts to extend the MSSM to accommodate the Higgs mass. In such extensions, new states interact with the Higgs, raising its mass by increasing the strength of the quartic interaction of the scalar potential. If the new states are integrated out supersymmetrically, their effects decouple and the Higgs mass is not increased. On the other hand, SUSY breaking can lead to non-decoupling effects that increase the Higgs mass. One possibility is a non-decoupling $F$ term, as in the NMSSM (MSSM plus a singlet) $[8,9]$ or $\lambda$ SUSY (allowing for a Landau pole) [10]. A second possibility is a non-decoupling $D$-term that results if the Higgs is charged under a new gauge group [11]. In general, these extensions require new states at the few hundred $\mathrm{GeV}$ scale, so that the new sources of SUSY breaking do not spoil naturalness.

For example, consider the NMSSM, where a singlet superfield, $S$, interacts with the MSSM Higgses, $H_{u, d}$, through the superpotential,

$$
W \supset \lambda S H_{u} H_{d}+\frac{M}{2} S^{2}+\mu H_{u} H_{d} .
$$


The $F$-term of $S$ gives

$$
V \supset\left|F_{S}\right|^{2}=\left|\lambda H_{u} H_{d}+M S\right|^{2}
$$

It generates additional Higgs quartic terms which potentially increase the Higgs mass. The potential is

$$
\Delta V=\lambda^{2}\left|H_{u} H_{d}\right|^{2}-\frac{(\lambda M)^{2}}{M^{2}+m_{S}^{2}}\left|H_{u} H_{d}\right|^{2}
$$

and we can understand it diagrammatically by Fig. 1 .
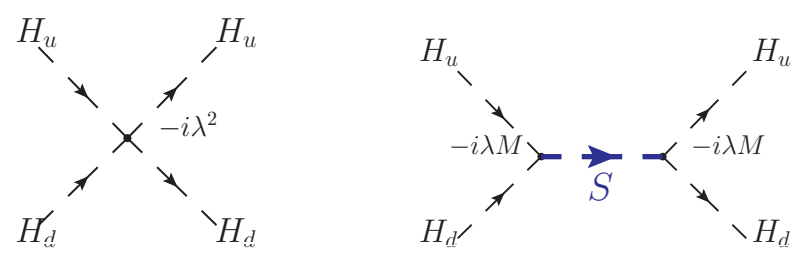

Figure 1: Additional Higgs quartic terms

The Higgs mass is increased by,

$$
\Delta m_{h}^{2}=\lambda^{2} v^{2} \sin ^{2} 2 \beta\left(\frac{m_{S}^{2}}{M^{2}+m_{S}^{2}}\right)
$$

where $m_{S}^{2}$ is the SUSY breaking soft mass $m_{S}^{2}|S|^{2}, \tan \beta=v_{u} / v_{d}$ is the ratio of the VEVs of the up and down-type Higgses, and $v=\sqrt{v_{u}^{2}+v_{d}^{2}}=174 \mathrm{GeV}$. Notice that this term decouples in the supersymmetric limit, $M \gg m_{S}$, which means $m_{S}$ should not be too small. On the other hand, $m_{S}$ feeds into the Higgs soft masses, $m_{H_{u, d}}^{2}$ at one-loop,

$$
\mu \frac{d m_{H_{u, d}^{2}}}{d \mu} \supset \frac{\lambda^{2} m_{S}^{2}}{8 \pi^{2}}
$$

requiring fine-tuning if $m_{S} \gg m_{h}$. The soft mass of $S$, that is $m_{S}^{2} \theta^{2} \bar{\theta}^{2} S^{\dagger} S$, gives $m_{H_{u}}^{2}$ along with a logarithmic divergence, and we have Eq.(1.7) as a consequence. Therefore, there is tension between raising the Higgs mass, which requires large $m_{S}$, and naturalness, which demands small $m_{S}$. Of course, in the limit of $m_{S} \rightarrow 0$, similar tension which is discussed in the beginning exists with respect to $m_{\tilde{t}}$.

Here, we point out that, contrary to the above example, a lack of light scalars can help raise the Higgs mass without a cost to naturalness, if the singlet has a Dirac mass.

\section{The Model}

we consider a modification of Eq. 1.3 where $S$ receives a Dirac mass with another singlet, $\bar{S}$,

$$
W=\lambda S H_{u} H_{d}+M S \bar{S}+\mu H_{u} H_{d} .
$$

We call this model the Dirac NMSSM. 


\subsection{Lagrangian of Dirac NMSSM}

From the spurion analysis the superpotential of Higgs sector in the Dirac NMSSM is given by

$$
W_{\text {Dirac }}=\lambda S H_{u} H_{d}+M S \bar{S}+\mu H_{u} H_{d}+c_{\bar{S}} \mu M \bar{S},
$$

and the corresponding potential is

$$
\begin{aligned}
V_{\text {Dirac }}= & \left|F_{\bar{S}}\right|^{2}+\left|F_{S}\right|^{2}+\left|F_{H_{u}}\right|^{2}+\left|F_{H_{d}}\right|^{2} \\
= & \left|\lambda H_{u} H_{d}+M S\right|^{2}+\left|M S+c_{\bar{S}} \mu M\right|^{2} \\
& +\left|(\lambda S+\mu) H_{d}\right|^{2}+\left|(\lambda S+\mu) H_{u}\right|^{2}
\end{aligned}
$$

where terms involving quark and lepton are omitted. The following soft supersymmetry breaking terms are allowed by the symmetries,

$$
\begin{aligned}
V_{\text {Dirac }}^{\text {soft }}= & m_{H_{u}}^{2}\left|H_{u}\right|^{2}+m_{H_{d}}^{2}\left|H_{d}\right|^{2}+m_{S}^{2}|S|^{2}+\left.m_{\bar{S}}^{2} \bar{S}\right|^{2} \\
& +\lambda A_{\lambda} S H_{u} H_{d}+M B_{S} S \bar{S}+\mu B H_{u} H_{d}+c . c . \\
& +t_{\bar{S}} \bar{S}+t_{S} S+c . c .
\end{aligned}
$$

The last tadpole arises from a non-holomorphic term $\mu^{\dagger} S$. Both soft tadpoles naturally have weakscale sizes due to the symmetry and spurion structure. As described later, the spectrum we consider is one shown in Fig. 2

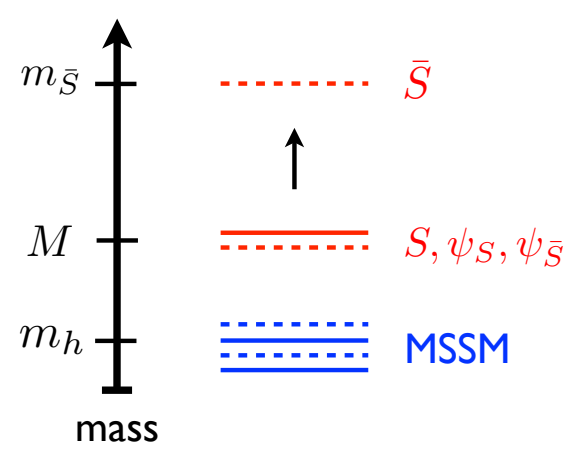

Figure 2: Schematic mass spectrum we consider in the Dirac NMSSM.

\section{Raising the Higgs Mass without Fine-tuning}

\subsection{Non-Decoupling Effects}

We would like to understand whether the new quartic term, $\left|\lambda H_{u} H_{d}\right|^{2}$, can naturally raise the Higgs mass. When we integrate out the $S$ and $\bar{S}$ chiral multiplets, normally we expect that the quartic potential decouples in the limit of heavy singlets. However, we find the $S$ and $\bar{S}$ exchanges 

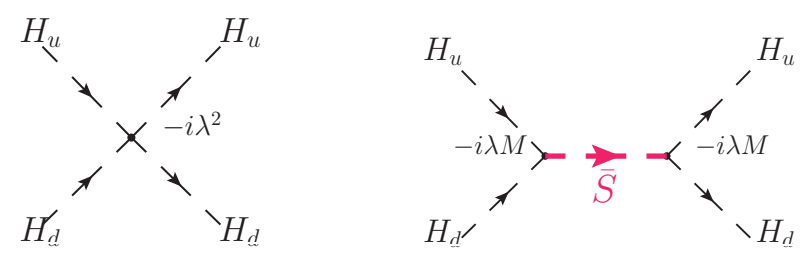

Figure 3: Non-decoupling effects in Dirac NMSSM
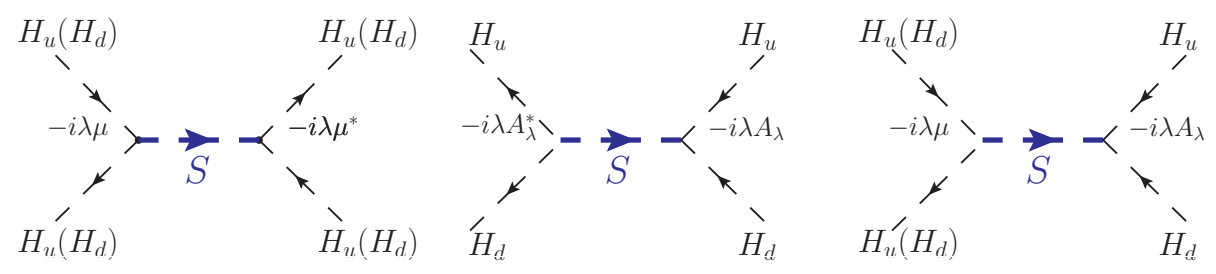

Figure 4: Other Higgs quartic terms in Dirac NMSSM

do not cancel the quartic term,

$$
\begin{aligned}
V_{e f f} & =\left|\lambda H_{u} H_{d}\right|^{2}\left(1-\frac{M^{2}}{M^{2}+m_{\bar{S}}^{2}}\right) \\
& -\frac{\lambda^{2}}{M^{2}+m_{S}^{2}}\left|A_{\lambda} H_{u} H_{d}+\mu^{*}\left(\left|H_{u}\right|^{2}+\left|H_{u}\right|^{2}\right)\right|^{2} .
\end{aligned}
$$

where we keep leading $\left(M^{2}+m_{S, \bar{S}}^{2}\right)^{-1}$ terms and neglect the tadpole terms for simplicity. The new contribution to the Higgs quartic does not decouple when $m_{\bar{S}}^{2}$ is large. The SM-like Higgs mass becomes,

$$
\begin{aligned}
m_{h}^{2}= & m_{h, \mathrm{MSSM}}^{2}\left(m_{\tilde{t}}\right)+\lambda^{2} v^{2} \sin ^{2} 2 \beta\left(\frac{m_{\bar{S}}^{2}}{M^{2}+m_{\bar{S}}^{2}}\right) \\
& -\frac{\lambda^{2} v^{2}}{M^{2}+m_{S}^{2}}\left|A_{\lambda} \sin 2 \beta-2 \mu^{*}\right|^{2},
\end{aligned}
$$

in the limit where the VEVs and mass-eigenstates are aligned, $H_{u} \rightarrow v_{u}+h \sin \beta$ and $H_{d} \rightarrow v_{d}+$ $h \cos \beta$. The second term, coming from diagrams of Fig. 3, shows so-called non-decoupling effect which is maximized by large $m_{\bar{S}}$. The second line of Eq.(3.2) can be understood by diagrams of Fig. 4, and it always reduces the size of quartic coupling.

By the way, in a limit of $M \ll m_{\bar{S}}$, the non-decoupling effect is easily derived by integrating out of $S$ and $\bar{S}$,

$$
\begin{aligned}
\int d^{4} \theta\left(1-m_{\bar{S}}^{2} \theta^{4}\right) \bar{S}^{\dagger} \bar{S} & +S^{\dagger} S+\left(\int d^{2} \theta \lambda S H_{u} H_{d}+M \bar{S} S+\text { h.c. }\right) \\
& \rightarrow \int d^{4} \theta \frac{\lambda^{2}\left(1-m_{\bar{S}}^{2} \theta^{4}\right)}{M^{2}}\left(H_{u} H_{d}\right)^{\dagger}\left(H_{u} H_{d}\right) .
\end{aligned}
$$




\subsection{Renormalization Group Equations}

The Higgs sector is natural when there are no large radiative corrections to $m_{H_{u, d}}^{2}$. The renormalization group (RG) of the up-type Higgs contains the terms,

$$
\begin{aligned}
\mu \frac{d}{d \mu} m_{H_{u}}^{2}= & \frac{1}{8 \pi^{2}}\left\{3 y_{t}^{2}\left(m_{\tilde{Q}_{3}}^{2}+m_{\tilde{t}_{R}}^{2}+m_{H_{u}}^{2}+\left|A_{t}\right|^{2}\right)\right. \\
& +\lambda^{2}\left(m_{S}^{2}+m_{H_{u}}^{2}+m_{H_{d}}^{2}+\left|A_{\lambda}\right|^{2}\right) \\
& \left.-3 g_{2}^{2} M_{2}^{2}-g_{1}^{2} M_{1}^{2}\right\}, \\
\mu \frac{d}{d \mu} m_{H_{d}}^{2}= & \frac{1}{8 \pi^{2}}\left\{\lambda^{2}\left(m_{S}^{2}+m_{H_{u}}^{2}+m_{H_{d}}^{2}+\left|A_{\lambda}\right|^{2}\right)\right. \\
& \left.-3 g_{2}^{2} M_{2}^{2}-g_{1}^{2} M_{1}^{2}\right\} .
\end{aligned}
$$

While heavy stops or $m_{S}^{2}$ lead to fine-tuning, we find that $m_{\bar{S}}^{2}$ does not appear. In fact, the RGs for $m_{H_{u, d}}^{2}$ are independent of $m_{\bar{S}}^{2}$ to all orders in mass-independent schemes, for example $\overline{M S}$ and $\overline{D R}$ schemes, This is clarified by dimensional analysis.

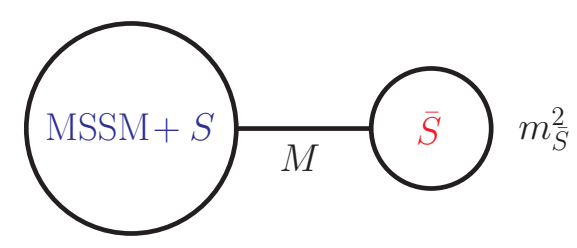

First of all, because $\bar{S}$ couples to the MSSM $+S$ sector only through the dimensionful coupling $M$, their interplay vanishes in $M \rightarrow 0$, and then terms involving $m_{\bar{S}}^{2}$ must proportional to $M$. Next, for the $U_{\bar{S}}$ conservation, a combination of lowest mass dimension is $|M|^{2} m_{\bar{S}}^{2}$, which has too high mass dimension to enter RG equations of the Higgs parameters, $m_{H_{u}}^{2}, m_{H_{d}}^{2}$ and $\mu B$, whose mass dimension is two. Hence, the large $m_{\bar{S}}$ does not upset naturalness through RGs.

\subsection{Threshold corrections}

We consider threshold corrections in the effective theory where the scalar component of $\bar{S}$ is integrated for $m_{\bar{S}} \gg M, \mu, m_{S}, m_{H_{u, d}}, A_{\lambda} \ldots$, and see if important corrections appear for $m_{H_{u, d}}^{2}$. One can see that the double insertion of $M$ is needed for $\bar{S}$ to involve $m_{H_{u, d}}^{2}$ as shown in a supergraph of Fig. 5 and consequently there is no quadratic sensitivity to $m_{\bar{S}}$. The soft mass $m_{\bar{S}}$ changes only finite piece of log-divergence. Hence there is only logarithmic sensitivity to $m_{\bar{S}}^{2}$ from the one-loop finite threshold correction,

$$
\delta m_{H}^{2} \equiv \delta m_{H_{u, d}}^{2}=\frac{(\lambda M)^{2}}{(4 \pi)^{2}} \log \frac{M^{2}+m_{\bar{S}}^{2}}{M^{2}} .
$$

which still allows for very heavy $m_{\bar{S}}^{2}$ without fine-tuning.

One may wonder if there are dangerous finite threshold corrections to $m_{H_{u}}^{2}$ at higher order, after integrating out $\bar{S}$. In fact, there is no quadratic sensitivity to $m_{\bar{S}}^{2}$ to all orders. This follows because any dependence on $m_{\bar{S}}^{2}$ must be proportional to $|M|^{2}$ (since $\bar{S}$ decouples when $M \rightarrow 0$ and by conservation of $\left.U(1)_{\bar{S}}\right)$, but $|M|^{2} m_{\bar{S}}^{2}$ has too high mass dimension. The mass dimension cannot be 


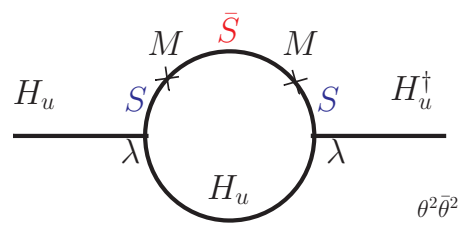

Figure 5: Supergraph of $m_{H_{u}}^{2}$ correction

reduced from other mass parameters appearing in the denominator because threshold corrections are always analytic functions of IR mass parameters [12]. This fact is from the matching of IR singularities. The full theory and the effective theory where the high energy dynamics down to a certain energy scale, e.g., $m_{\bar{S}}$, is integrated out must have same IR structure, that is, IR singularities are same in the both theories. If there is threshold corrections which is not analytic for IR mass parameters, such as,

$$
\delta m^{2} \sim \frac{|M|^{2} m_{\bar{S}}^{2}}{m_{\mathrm{IR}}^{2}},
$$

they will be new IR singularities when $m_{\mathrm{IR}}^{2} \rightarrow 0$, which contradicts the IR matching.

\subsection{Semi-soft SUSY breaking}

It may seem contradictory that naturalness is maintained in the limit of very heavy $m_{\bar{S}}$, since removing the $\bar{S}$ scalar from the spectrum constitutes a hard breaking of SUSY. In the effective theory with $m_{\bar{S}} \rightarrow \infty$, there exist the $\bar{S}$ fermion with a Dirac mass and several terms originating from $F_{\bar{S}}$,

$$
\mathscr{L} \supset i \bar{\psi}_{\bar{S}} \bar{\sigma}^{\mu} \partial_{\mu} \psi_{\bar{S}}-M \psi_{S} \psi_{\bar{S}}-M^{2}\left|S+c_{\bar{S}} \mu\right|^{2}
$$

as well as terms derived by superfield,

$$
\begin{gathered}
\mathscr{K} \supset H_{u}^{\dagger} H_{u}+H_{d}^{\dagger} H_{d}+S^{\dagger} S, \\
W \supset \lambda S H_{u} H_{d}+\mu H_{u} H_{d} .
\end{gathered}
$$

This theory can be written in superfields and soft breakings if we reintroduce the scalar, $\phi_{\bar{S}^{\prime}}$, and $F$-term, $F_{\bar{S}^{\prime}}$, to form a chiral supermultiplet of

$$
\bar{S}^{\prime}=\phi_{\bar{S}^{\prime}}+\theta \psi_{\bar{S}}+\theta^{2} F_{\bar{S}^{\prime}}
$$

Eq.(3.9) is rewritten,

$$
\begin{aligned}
& \mathscr{K}_{\text {eff }}=\bar{S}^{\prime \dagger} \bar{S}^{\prime}-\theta^{2} \bar{\theta}^{2}\left(M^{2}\left|S+c_{\bar{S}} \mu\right|^{2}\right), \\
& W_{\text {eff }}=-\theta^{2}\left(M \mathscr{D}^{\alpha} S \mathscr{D}_{\alpha} \bar{S}^{\prime}\right)
\end{aligned}
$$

where $\phi_{\bar{S}^{\prime}}$ and $F_{\bar{S}^{\prime}}$ are completely decoupled from the other states. Therefore, the low energy effective theory even in absence of scalar of $\bar{S}$ is equivalent to a theory with only softly broken supersymmetry. We call this mechanism semi-soft supersymmetry breaking. It is crucial that $\bar{S}$ couples to the other fields only through dimensionful couplings. 


\section{Higgs Mass}

The most natural region of parameter space, summarized in Fig. 2, has $m_{S}$ and $M$ at the hundreds of $\mathrm{GeV}$ scale, to avoid large corrections to $m_{H_{u}}$, and large $m_{\bar{S}} \gtrsim 10 \mathrm{TeV}$, to maximize the second term of Eq. (3.2). The tree-level contribution to the Higgs mass can be large enough such that $m_{\tilde{t}}$ takes a natural value at the hundreds of $\mathrm{GeV}$ scale.

\subsection{CP-Even Neutral Scalars}

For simplicity we assume there are no $\mathrm{CP}$ violations in the following studies. The minimization conditions for the Higgs and Singlet scalars lead

$$
\begin{aligned}
& \mu_{e f f}^{2}+m_{H_{d}}^{2}-b_{e f f} \tan \beta+\frac{m_{Z}^{2}}{2} \cos 2 \beta+\lambda^{2} v^{2} \sin ^{2} \beta=0, \\
& \mu_{e f f}^{2}+m_{H_{u}}^{2}-b_{e f f} \cot \beta-\frac{m_{Z}^{2}}{2} \cos 2 \beta+\lambda^{2} v^{2} \cos ^{2} \beta=0, \\
& M^{2}+m_{S}^{2}+\frac{\lambda v^{2}}{v_{S}}\left(\mu+\lambda v_{S}\right) \\
& +\frac{1}{v_{S}}\left(M B_{S} v_{\bar{S}}-\frac{\lambda^{2} A_{\lambda} v^{2}}{2} \sin 2 \beta+c_{\bar{S}} M^{2} \mu+t_{s}\right)=0, \\
& M^{2}+m_{\bar{S}}^{2}+\frac{1}{v_{\bar{S}}}\left(M B_{S} v_{S}-\frac{\lambda M v^{2}}{2} \sin 2 \beta+t_{\bar{S}}\right)=0 .
\end{aligned}
$$

where $v_{S}=\langle S\rangle$ and $v_{\bar{S}}=\langle\bar{S}\rangle$, and it is convenient to use

$$
\begin{aligned}
& b_{\text {eff }} \equiv \mu B+\lambda\left(A_{\lambda} v_{S}+M v_{\bar{S}}\right), \\
& \mu_{\text {eff }} \equiv \mu+\lambda v_{S}, \\
& \bar{m}_{A}^{2} \equiv b_{\text {eff }} /\left(s_{\beta} c_{\beta}\right) .
\end{aligned}
$$

The mass matrix of CP-even neutral scalars at tree level is given by $\mathscr{M}_{H^{0}, i j}^{2}\left(=\mathscr{M}_{H^{0}, j i}^{2}\right)$ in the base of $\operatorname{Re}\left(H_{d}^{0}, H_{u}^{0}, S, \bar{S}\right)^{T} / \sqrt{2}$ where

$$
\begin{aligned}
& \mathscr{M}_{H^{0}}^{2}=
\end{aligned}
$$

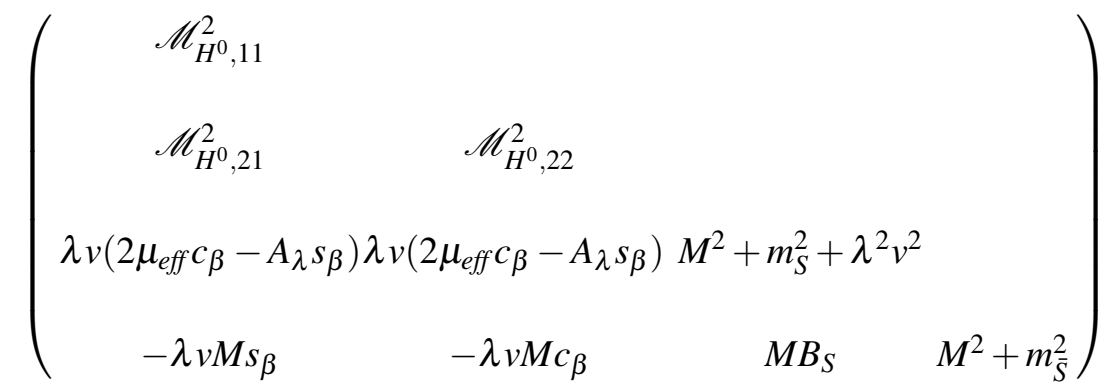

and where

$$
\begin{aligned}
\mathscr{M}_{H^{0}, 11}^{2} & =\bar{m}_{A}^{2} s_{\beta}^{2}+m_{Z}^{2} c_{\beta}^{2}, \\
\mathscr{M}_{H^{0}, 22}^{2} & =\bar{m}_{A}^{2} c_{\beta}^{2}+m_{Z}^{2} s_{\beta}^{2}, \\
\mathscr{M}_{H^{0}, 21}^{2} & =-\left(\bar{m}_{A}^{2}+m_{Z}^{2}-2 \lambda^{2} v^{2}\right) s_{\beta} c_{\beta} .
\end{aligned}
$$


Here $s_{\beta}\left(c_{\beta}\right)$ denotes $\sin \beta(\cos \beta)$, and the vacuum conditions of Eqs.(4.1)(4.2) are used for $\mathscr{M}_{H^{0}, 11}^{2}$ and $\mathscr{M}_{H^{0}, 22}^{2}$. Note that non-decoupling effect can be seen in $\mathscr{M}_{H^{0}, 21}^{2}$, which completely remains when $m_{\bar{S}} \rightarrow \infty$.

\section{Radiative Corrections}

We take into account radiative corrections to the mass matrix, and an estimation of the stop mass is particularly important. The one-loop level calculation of $y_{t}$ is easy to perform, which raises the SM-like Higgs mass, but it is well-known that the two-loop contributions tend to reduce the Higgs mass. Since only one-loop calculation leads optimistic result about naturalness, we adopt RG-improved one-loop calculation which is consistent enough with two-loop calculation of the MSSM. The similar result is given by Ref.[13].

\subsection{One loop corrections}

The corrections are included by two parts. One is at $\mathscr{O}\left(y_{t}^{4}\right)$ calculated by effective potential, and the other is at $\mathscr{O}\left(y_{t}^{2} g^{\prime 2}, y_{t}^{2} g_{2}^{2}\right)$ involving wave function renormalization of $\mathscr{O}\left(y_{t}^{2}\right)$. The effective potential at one-loop is given by

$$
\begin{aligned}
& \Delta V^{(1)}(H, Q)= \\
& \frac{3}{32 \pi^{2}}\left\{m_{\tilde{t}_{1}}^{4}(H)\left(\log \frac{m_{\tilde{t}_{1}}^{2}(H)}{Q^{2}}-\frac{3}{2}\right)+m_{\tilde{t}_{2}}^{4}(H)\left(\log \frac{m_{\tilde{t}_{2}}^{2}(H)}{Q^{2}}-\frac{3}{2}\right)\right. \\
& \left.\quad-2 m_{t}^{4}(H)\left(\log \frac{m_{t}^{2}(H)}{Q^{2}}-\frac{3}{2}\right)\right\},
\end{aligned}
$$

where the mass parameters are $H_{u, d}$ fields dependent,

$$
\begin{aligned}
& m_{t}^{2}(H)=\left|y_{t} H_{u}\right|^{2}, \\
& m_{\tilde{t}_{1}}^{2}(H)=m_{\tilde{t}}^{2}+\left|y_{t} H_{u}^{0}\right|^{2}-\left|y_{t} \mu H_{d}^{0}\right|, \\
& m_{\tilde{t}_{2}}^{2}(H)=m_{\tilde{t}}^{2}+\left|y_{t} H_{u}^{0}\right|^{2}+\left|y_{t} \mu H_{d}^{0}\right| .
\end{aligned}
$$

Here we assume $A_{t}=0$ and $m_{\tilde{t}_{R}}^{2}=m_{\tilde{Q}_{3}\left(\tilde{t}_{L}\right)}^{2}=m_{\tilde{t}}^{2}$ for simplicity, and we adopt $Q$ as a renormalization scale instead of $\mu$ to avoid confusion with a Lagrangian parameter.

In addition, the corrections of $\mathscr{O}\left(y_{t}^{2} g^{2}, y_{t}^{2} g_{2}^{2}\right)$ require wave function renormalization as well as vertex corrections. Since both corrections are from the same one-loop supergraph that the fermionic part enters wave function renormalization and the bosonic part enters vertex correction as seen Fig. 6, their divergences are canceled leaving finite corrections, and we match them onto the Higgs potential,

$$
\Delta V^{(2)}(H)=-\frac{g^{\prime 2}+g_{2}^{2}}{4} \frac{3 y_{t}^{2}}{8 \pi^{2}} \ln \frac{m_{\tilde{t}}^{2}+m_{t}^{2}}{m_{t}^{2}}\left(\left|H_{u}^{0}\right|^{4}-\frac{1}{2}\left|H_{u}^{0}\right|^{2}\left|H_{d}^{0}\right|^{2}\right)
$$

where we neglected the mixing between $\tilde{t}_{R}$ and $\tilde{t}_{L}$. 

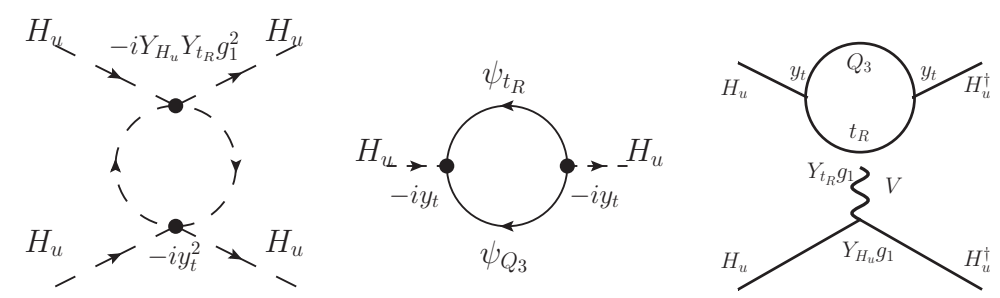

Figure 6: Left: $\mathscr{O}\left(y_{t}^{2} g^{\prime 2}\right)$ vertext correction, center: wave function renormalization leading corrections of $\mathscr{O}\left(y_{t}^{2} g^{2}\right)$. Right: One-loop supergraph. One without attachment of gauge field leads wavefunction renormalization, and one with gauge field leads vertex corrections of left .

For the new potential at one-loop level, the vacuum conditions for $H_{u, d}$ are shifted,

$$
\begin{aligned}
& \mu_{\text {eff }}^{2}+m_{H_{d}}^{2}-b_{\text {eff }} \tan \beta+\frac{m_{Z}^{2}}{2} \cos 2 \beta+\lambda^{2} v^{2} \sin ^{2} \beta+\left\langle\frac{1}{2 H_{d}^{0}} \frac{\partial \Delta V}{\partial H_{d}^{0}}\right\rangle=0, \\
& \mu_{\text {eff }}^{2}+m_{H_{u}}^{2}-b_{\text {eff }} \cot \beta-\frac{m_{Z}^{2}}{2} \cos 2 \beta+\lambda^{2} v^{2} \cos ^{2} \beta+\left\langle\frac{1}{2 H_{u}^{0}} \frac{\partial \Delta V}{\partial H_{u}^{0}}\right\rangle=0 .
\end{aligned}
$$

Hence, the mass matrix of Eq.(4.8) is modified by

$$
\begin{aligned}
\Delta \mathscr{M}_{H^{0}, 11}^{2} & =\frac{1}{2}\left\langle\frac{\partial^{2} \Delta V}{\partial\left(H_{d}^{0}\right)^{2}}\right\rangle-\left\langle\frac{1}{2 H_{d}^{0}} \frac{\partial \Delta V}{\partial H_{d}^{0}}\right\rangle \\
& =\frac{3}{32 \pi^{2}}\left(\left(y_{t} \mu\right)^{2}-\frac{y_{t} \mu\left(m_{\tilde{t}}^{2}+m_{t}^{2}\right)}{v c_{\beta}} \log \frac{m_{\tilde{t}_{1}}^{2}}{m_{\tilde{t}_{2}}^{2}}\right), \\
\Delta \mathscr{M}_{H^{0}, 22}^{2} & =\frac{1}{2}\left\langle\frac{\partial^{2} \Delta V}{\partial\left(H_{u}^{0}\right)^{2}}\right\rangle-\left\langle\frac{1}{2 H_{u}^{0}} \frac{\partial \Delta V}{\partial H_{u}^{0}}\right\rangle \\
& =\frac{3 y_{t}^{2} m_{t}^{2}}{8 \pi^{2}} \ln \frac{m_{\tilde{t}_{1}}^{2} m_{\tilde{t}_{2}}^{2}}{m_{t}^{4}}-m_{Z}^{2} s_{\beta}^{2}\left(\frac{3 y_{t}^{2}}{8 \pi^{2}} \ln \frac{m_{\tilde{t}}^{2}+m_{t}^{2}}{m_{t}^{2}}\right), \\
\Delta \mathscr{M}_{H^{0}, 21}^{2} & =\frac{1}{2}\left\langle\frac{\partial^{2} \Delta V}{\partial H_{u}^{0} \partial H_{d}^{0}}\right\rangle \\
& =\frac{3 y_{t}^{2} m_{t} \mu}{16 \pi^{2}} \log \frac{m_{\tilde{t}_{1}}^{2}}{m_{\tilde{t}_{2}}^{2}}+m_{Z}^{2} s_{\beta} c_{\beta}\left(\frac{3 y_{t}^{2}}{16 \pi^{2}} \ln \frac{m_{\tilde{t}}^{2}+m_{t}^{2}}{m_{t}^{2}}\right) .
\end{aligned}
$$

In Eqs.(5.9)(5.10), the vacuum shifts must be considered since the vacuum conditions are already used in Eq.(4.8). This result is consistent with Refs.[13].

\section{Benchmark Parameters and Stop Mass}

When we estimate the stop mass in the Dirac NMSSM, we take $M$ and $m_{\bar{S}}$ to be free parameters since $M$ is common in both models and $m_{\bar{S}}$ is essential to discuss the non-decoupling effect. Other parameters are fixed according to the table of benchmark parameters. We have chosen $\lambda$ to saturate 
$m_{\tilde{t}}[\mathrm{GeV}]$

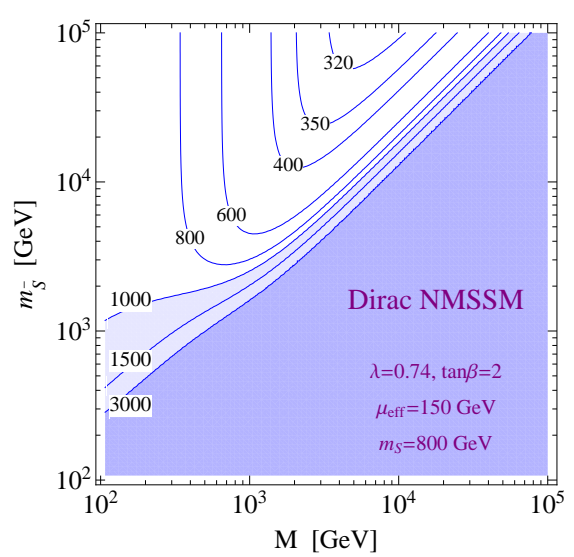

$m_{\tilde{t}}[\mathrm{GeV}]$

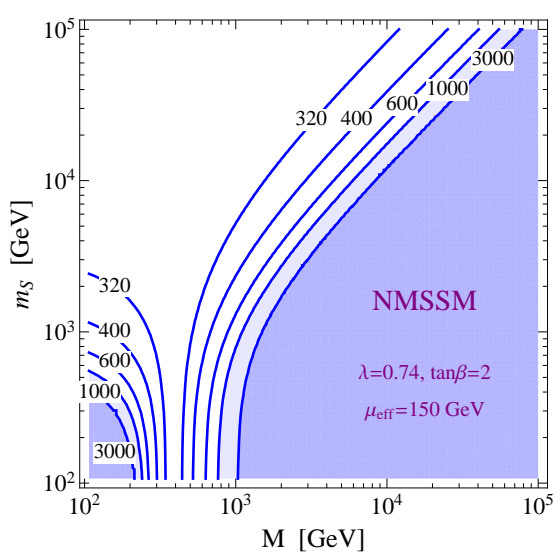

Figure 7: Stop soft mass in the Dirac NMMSM (left) and NMMSM (right).

the upper-limit such that it does not reach a Landau pole below the unification scale [14]. For the NMSSM, we treat $m_{S}$ as a free parameter instead of $m_{\bar{S}}$ and must use the different $b_{\text {eff }}$,

$$
b_{e f f} \equiv \mu B+\lambda v_{S}\left(A_{\lambda}+M\right)
$$

The other parameters are as same as in the Dirac NMSSM.

$$
\begin{array}{ccc}
\lambda=0.74 & \tan \beta=2 & \mu_{\text {eff }}=150 \mathrm{GeV} \\
b_{\text {eff }}=(190 \mathrm{GeV})^{2} & A_{\lambda}=0 & B_{s}=100 \mathrm{GeV} \\
m_{S}=800 \mathrm{GeV} \\
\hline
\end{array}
$$

Table 1: Benchmark parameters

One may worry about the size of singlet VEVs especially from the tadpoles because we fixed $\mu_{\text {eff }}$ and $b_{\text {eff }}$ defined in Eqs.(4.5,4.6). However we can see the VEVs adequately small,

$$
\begin{aligned}
& v_{S} \simeq \frac{\left(c_{\bar{S}}^{*} \mu^{*}|M|^{2}+t_{S}^{*}\right)\left(|M|^{2}+m_{\bar{S}}^{2}\right)}{\left(|M|^{2}+m_{S}^{2}\right)\left(|M|^{2}+m_{\bar{S}}^{2}\right)} \sim \mu^{*} \\
& v_{\bar{S}} \simeq \frac{t_{\bar{S}}^{*}\left(|M|^{2}+m_{S}^{2}\right)}{\left(|M|^{2}+m_{S}^{2}\right)\left(|M|^{2}+m_{\bar{S}}^{2}\right)} \sim \frac{(\mu M)^{*} m_{\bar{S}}}{|M|^{2}+m_{\bar{S}}^{2}}
\end{aligned}
$$

where the tadpoles scale as $t_{S} \sim \mu^{*} m_{S}^{2}$ and $t_{\bar{S}} \sim \mu M m_{\bar{S}}$.

We now estimate the stop mass. For each value of $\left(M, m_{\bar{S}}\right)$, the stop soft masses, $m_{\tilde{t}}=m_{\tilde{t}_{R}}=$ $m_{\tilde{Q}_{3}}$, are chosen to maintain the lightest scalar mass at $125 \mathrm{GeV}$. As results are shown in Fig. 7, basically the stop mass becomes small as $m_{\bar{S}}$ increases for the fixed $M$ because more non-decoupling effect remains. Hence, the natural region should spread in large $m_{\bar{S}}$ where the naturalness is not suffered from fine-tuning thanks to the semi-soft breaking. However, this argument does not apply for the low $M$ region. This is because the $S$ exchange effect, which is given by the second line of Eq.(3.2), is enhanced and decreases the tree-level Higgs mass when $M$ is small. 


\section{Naturalness}

In this section, the naturalness is evaluated. There are several measures, and we take a bottomup approach described in Ref. [15] since we do not specify any UV completions and discuss based on low-energy parameters.

The degree of fine-tuning is estimated by

$$
\Delta=\frac{2}{m_{h}^{2}} \max \left(m_{H_{u}}^{2}, m_{H_{d}}^{2}, \frac{d m_{H_{u}}^{2}}{d \ln \mu} L, \frac{d m_{H_{d}}^{2}}{d \ln \mu} L, \delta m_{H}^{2}, b_{e f f}\right),
$$

where $L \equiv \log \left(\Lambda_{\text {mess }} / m_{\tilde{t}}\right)$, and $\Lambda_{\text {mess }}$ is a scale at which particle masses are generated. We take two values of $\Lambda_{\text {mess }}$ corresponding to low-scale $(L=6)$ and high-scale $(L=30)$ SUSY breaking. corresponding to low-scale SUSY breaking. We assume that contributions through gauge couplings to the RGs for $m_{H_{u, d}}^{2}$ are subdominant. To be more concrete about Eq. (7.1), the RGE effects to $m_{H_{u}}$, for example, are separated by each parameter,

$$
\frac{d m_{H_{u}}^{2}}{d \ln \mu} L=\left(\frac{3 y_{t}^{2} m_{\tilde{Q}_{3}}^{2}}{8 \pi^{2}} L, \frac{3 y_{t}^{2} m_{\tilde{t}_{R}}^{2}}{8 \pi^{2}} L, \frac{\lambda^{2} m_{S}^{2}}{8 \pi^{2}} L \ldots\right) .
$$

The results are shown in Fig. 8.

In the Drac NMSSM, the degree of tuning $\Delta$ is mostly determined by the RGE effect from $m_{\tilde{t}}$, and when $\mathrm{M}$ is quit large, $M>1 \mathrm{TeV}, \Delta$ is determined by the threshold correction given in Eq. (3.7). Actually as shown in Fig. 8, the shape of curves almost corresponds to that of stop mass shown in Fig. 7, and the upper-right region is dominated by the threshold correction. The difference between low- and high-scale SUSY breaking cases is basically the difference of absolute value by a factor of 5 . The tuning in the NMSSM is determined by the RGE effects not only through $m_{\tilde{t}}$ but also $m_{S}$.

We see that the least-tuned region of the Dirac NMSSM corresponds to $M \sim 2 \mathrm{TeV}$ and $m_{\bar{S}} \gtrsim 10 \mathrm{TeV}$, where the tree-level correction to the Higgs mass is maximized. The fact that the large SUSY breaking, $m_{\bar{S}}$, leads to more natural theory is counter-intuitive, but, thanks to the mechanism introduced as semi-soft SUSY breaking, this particular SUSY breaking is irrelevant to the naturalness. On the other-hand, the NMSSM becomes highly tuned when $m_{S}$ is large, and then $m_{S} \lesssim 500 \mathrm{GeV}$ is favored. Note that region of low-tuning in the NMSSM extends to the supersymmetric limit, $m_{S} \rightarrow 0$. In this region the Higgs mass is increased by a new contribution to the quartic coupling proportional to $\lambda^{2}\left(M \mu \sin 2 \beta-\mu^{2}\right) / M^{2}$ (see Ref.[9] for more details).

\subsection{Summary}

The LHC has discovered a new particle, consistent with the Higgs boson, with a mass near $125 \mathrm{GeV}$. Weak-scale supersymmetry must be reevaluated in light of this discovery. Naturalness demands new dynamics beyond the minimal theory, such as a non-decoupling $F$-term, but this implies new sources of SUSY breaking that themselves threaten naturalness. We have identified a new model where the Higgs couples to a singlet field with a Dirac mass. The non-decoupling $F$-term is naturally realized through semi-soft SUSY breaking, because large $m_{\bar{S}}$ helps raise the Higgs mass but does not threaten naturalness. 

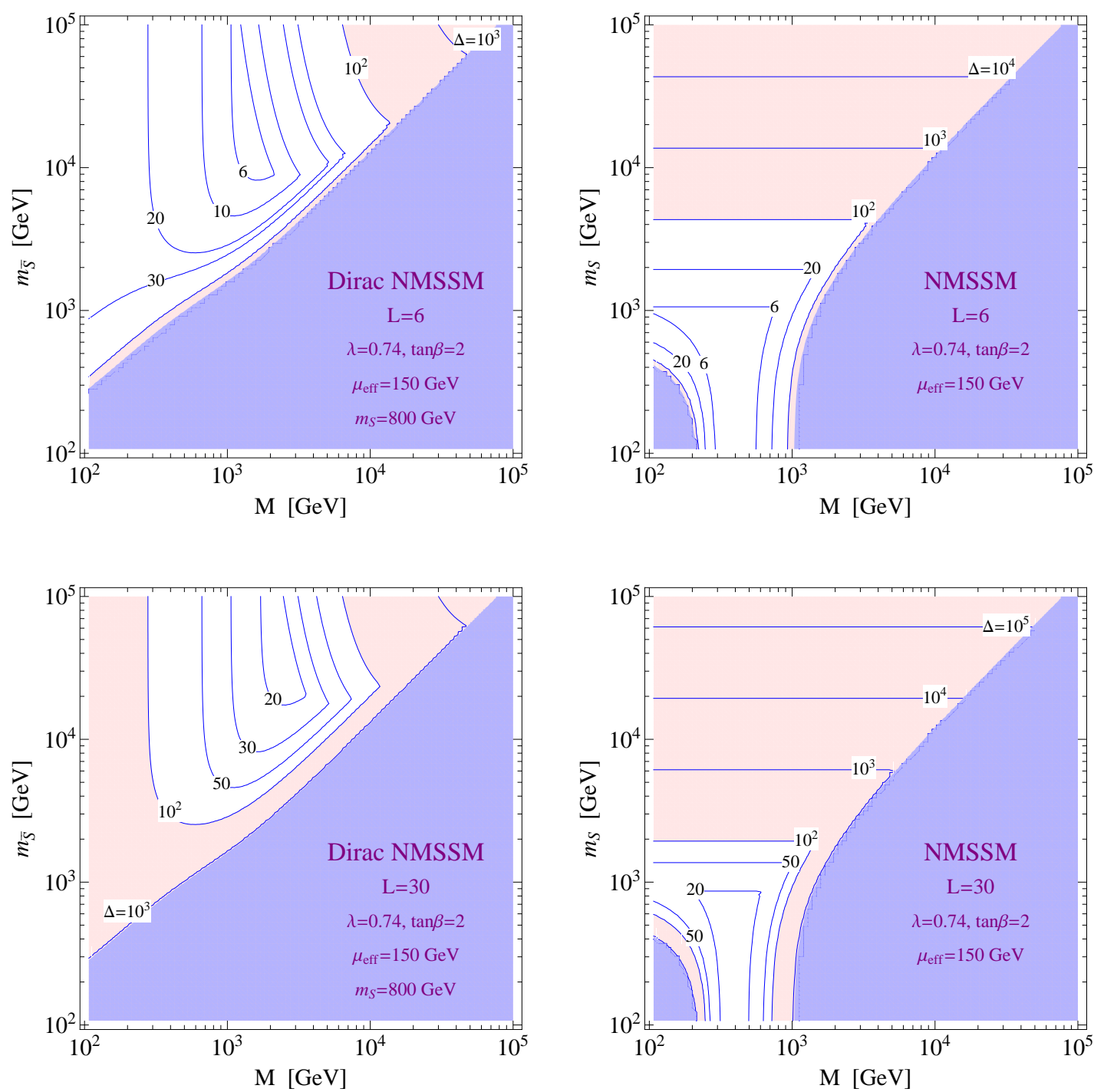

Figure 8: The tuning $\Delta$, defined in Eq. eq:tune, for the Dirac NMSSM is shown on the left as a function of $M$ and $m_{\bar{S}}$. For comparison, the tuning of the NMSSM is shown on the right, as a function of $M$ and $m_{S}$. The red region has high fine-tuning, $\Delta>100$, and the purple region requires $m_{\tilde{t}}>2 \mathrm{TeV}$, signaling severe fine-tuning $\gtrsim \mathscr{O}\left(10^{3}\right)$. The tuning is evaluated with $L=6(30)$ in the upper (lower) plots.

The key feature of semi-soft SUSY breaking in the Dirac NMSSM is that $\bar{S}$ couples to the MSSM only through the dimensionful Dirac mass, $M$. We noted that interactions between $\bar{S}$ and other new states are not constrained by naturalness, even if these states experience SUSY breaking. Therefore, the Dirac NMSSM represents a new type of portal, whereby our sector can interact with new sectors, with large SUSY breaking, without spoiling naturalness in our sector.

\section{References}


[1] G. Aad et al. [ATLAS Collaboration], Phys. Lett. B 716, 1 (2012), 1207.7214; S. Chatrchyan et al. (CMS Collaboration), Phys. Lett. B 716, 30 (2012), 1207.7235.

[2] L. J. Hall, D. Pinner and J. T. Ruderman, JHEP 1204, 131 (2012), 1112.2703.

[3] J. D. Wells, hep-ph/0306127;

[4] S. Dimopoulos and G. F. Giudice, Phys. Lett. B 357, 573 (1995) [hep-ph/9507282]; A. Pomarol and D. Tommasini, Nucl. Phys. B 466, 3 (1996) [hep-ph/9507462]; A. G. Cohen, D. B. Kaplan and A. E. Nelson, Phys. Lett. B 388, 588 (1996) [hep-ph/9607394]; M. Papucci, J. T. Ruderman and A. Weiler, JHEP 1209, 035 (2012) [arXiv:1110.6926 [hep-ph]]; C. Brust, A. Katz, S. Lawrence and R. Sundrum, JHEP 1203, 103 (2012) [arXiv:1110.6670 [hep-ph]].

[5] T. J. LeCompte and S. P. Martin, Phys. Rev. D 85, 035023 (2012) [arXiv:1111.6897 [hep-ph]];

[6] J. Fan, M. Reece and J. T. Ruderman, JHEP 1111, 012 (2011) [arXiv:1105.5135 [hep-ph]]; J. Fan, M. Reece and J. T. Ruderman, JHEP 1207, 196 (2012) [arXiv:1201.4875 [hep-ph]]; M. Baryakhtar, N. Craig and K. Van Tilburg, JHEP 1207, 164 (2012) [arXiv:1206.0751 [hep-ph]].

[7] For a review, R. Barbier, C. Berat, M. Besancon, M. Chemtob, A. Deandrea, E. Dudas, P. Fayet and S. Lavignac et al., Phys. Rept. 420, 1 (2005) [hep-ph/0406039]. For recent studies, see for example, C. Csaki, Y. Grossman and B. Heidenreich, Phys. Rev. D 85, 095009 (2012) [arXiv:1111.1239 [hep-ph]]. P. W. Graham, D. E. Kaplan, S. Rajendran and P. Saraswat, JHEP 1207, 149 (2012) [arXiv:1204.6038 [hep-ph]]. J. T. Ruderman, T. R. Slatyer and N. Weiner, arXiv:1207.5787 [hep-ph].

[8] J. R. Espinosa and M. Quiros, Phys. Lett. B 279, 92 (1992); U. Ellwanger, C. Hugonie and A. M. Teixeira, Phys. Rept. 496, 1 (2010) [arXiv:0910.1785 [hep-ph]];

[9] Y. Nomura, D. Poland and B. Tweedie, Phys. Lett. B 633, 573 (2006) [hep-ph/0509244]; A. Delgado, C. Kolda, J. P. Olson and A. de la Puente, Phys. Rev. Lett. 105, 091802 (2010) [arXiv:1005.1282 [hep-ph]];

[10] R. Harnik, G. D. Kribs, D. T. Larson and H. Murayama, Phys. Rev. D 70, 015002 (2004) [hep-ph/0311349]; R. Barbieri, L. J. Hall, Y. Nomura and V. S. Rychkov, Phys. Rev. D 75, 035007 (2007) [hep-ph/0607332];

[11] P. Batra, A. Delgado, D. E. Kaplan and T. M. P. Tait, JHEP 0402, 043 (2004) [hep-ph/0309149]; A. Maloney, A. Pierce and J. G. Wacker, JHEP 0606, 034 (2006) [hep-ph/0409127]; C. Cheung and H. L. Roberts, arXiv:1207.0234 [hep-ph].

[12] H. Georgi, Ann. Rev. Nucl. Part. Sci. 43, 209 (1993).

[13] H. E. Haber and R. Hempfling, Phys. Rev. D 48, 4280 (1993) [hep-ph/9307201]. M. S. Carena, J. R. Espinosa, M. Quiros and C. E. M. Wagner, Phys. Lett. B 355, 209 (1995) [hep-ph/9504316], H. E. Haber, R. Hempfling and A. H. Hoang, Z. Phys. C 75, 539 (1997) [hep-ph/9609331]. M. S. Carena, J. R. Ellis, A. Pilaftsis and C. E. M. Wagner, Nucl. Phys. B 586, 92 (2000) [hep-ph/0003180].

[14] R. Barbieri, L. J. Hall, A. Y. Papaioannou, D. Pappadopulo and V. S. Rychkov, JHEP 0803, 005 (2008) [arXiv:0712.2903 [hep-ph]].

[15] R. Kitano and Y. Nomura, Phys. Rev. D 73, 095004 (2006) [hep-ph/0602096]. 